\title{
Integrative approach to a difficult trichology patient
}

\author{
Natalie Barunova* \\ International Scientific-Practical Centre "Trichology”, Moscow, Russia
}

\begin{abstract}
Folliculitis decalvans belongs to a group of primary cicatricial alopecias with neutrophilic inflammation of the scalp. It is characterized by recurrent purulent follicular exudation with inevitable destruction of pilosebaceous unit as an outcome of the disease. Staphylococcus aureus is supposed to play an important role in the pathogenesis of the disease. The treatment is usually focused on the eradication of S. aureus.

A clinical case of effective adjuvant treatment of folliculitis decalvans patient is presented in this manuscript. The previous traditional treatment with antibiotics, topical glucocorticosteroids, oral prednisone and retinoid treatment had minor efficacy and subsequent recurrence. Integrative approach to this difficult case brought the patient into remission and improve the patient's condition.
\end{abstract}

\section{Introduction}

Scarring alopecias relate to a group of relatively rare diseases with one common feature - inevitable destruction of pilosebaceous unit due to replacement of hair follicles by fibrous tissue.

FD is classified as a primary neutrophilic scarring alopecia according to the classification from the 2001 Workshop on cicatricial alopecias at Duke University Medical Center [1].

It is characterized by recurrent purulent follicular exudation with patches of scarring (cicatricial) alopecia as an outcome of the condition and low efficacy of the treatment. The patients' frequent complaints are itching, pain and burning sensations.

The etiology of the disease is not clear, but Staphylococcus aureus is supposed to play an important role in in this disease as it is isolated from bacterial specimens in the vast majority of cases. The treatment is usually focused on the eradication of $S$. aureus $[2,3]$.

Folliculitis decalvans occurs in middle-aged adults and young people, males and females, with the lesions mainly occur in the vertex, and occipital areas. Clinically, the lesions present with perifollicular erythema and follicular pustules, yellow-gray scales, follicular tufts and lack of ostia, and often - erosions and hemorrhagic crusts, hyperkeratosis, starburst signs [4-6].

The repeated outbreaks of pustules result in the hair follicle destruction with the formation of the scarring alopecia patches. These patches may have oval or round shape, with ivory -white and whitepink colour.

The areas of follicular destruction may vary from extensive rapidly growing areas of hair loss to limited small scarring patches without disease progression over the time.

One of the common feature in FD is tufted hair follicular lesions, with several hairs (5-18) emerging from a single follicular opening.

Although it was discussed that tufted folliculitis could be a separate entity or a specific form of FD, at the same time it is well known that polytrichia can be seen as a secondary finding in other forms of scarring alopecias, such as dissecting cellulites, lichen planopilaris, discoid lupus erythematosus, central centrifugal cicatricial alopecia and acne keloidalis nuchae [2,7-9]. $[2,10]$ :

Differential diagnosis is performed with the following conditions

- Dissecting folliculitis - occurs almost exclusively in males. Clinical features include boggy scalp, deep inflammatory nodes, interconnected sinus tracts with purulent material;

- Acne keloidalis nuchae - also occurs mainly in males (predominantly in African-Americans), with nodes, papules and pustules located in the occipital area. Acne keloidalis results in hypertrophic scarring;

- Other scarring alopecias (discoid lupus erythematosus, pseudopelade of Brocq, central centrifugal cicatricial alopecia and lichen planopilaris) belong to a group of lymphocytic primary cicatricial alopecias. In contrast to FD they do not have pustules as primary lesions;

- $\quad$ Deep fungal scalp infection should be excluded by performing light microscopy and fungal culture;

- In its early stage folliculitis decalvans may be misdiagnosed as folliculitis simplex. In this case trichoscopy usually helps to reveal scarring patches with lacking follicle ostia.

\section{Clinical case}

Female patient, 30 y. o., presented to our clinic with the following complaints: pain and severe itching in the areas of skin lesions on the scalp, hair loss, and also - joint pain, bloating, epigastric pain, weight

Correspondence to: Natalie Barunova, MD, International Scientific-Practical Centre "Trichology”, Moscow, Russia, E-mail: nataligb@mail.ru

Key words: folliculitis decalvans, adjuvant therapy

Received: October 16, 2016; Accepted: November 05, 2016; Published: November 08, 2016 
gain, face and leg swelling.

\section{Anamnesis morbi}

The patient was followed up by dermatologists within 4 years with the diagnosis: Folliculitis decalvans.

Her previous treatment included antibiotics (rifampicin, clindamycin), prednisone, retinoid treatment - per os; mupirocin, fusidic acid, antifungal therapy, topical glucocorticosteroids - as local treatment. The treatment had minor efficacy with subsequent recurrence of the disease.

Her allergic history was compromised, she was complaining of the joints pain and had the following gastroenterological conditions detected: Gastroesophageal Reflux Disease (GERD), Irritable Bowel Syndrome (IBS), Small Intestinal Bacterial Overgrowth (SIBO).

The family history revealed scalp psoriasis and diabetes mellitus II among the family members.

\section{Patient's examination}

Trichoscopy and global photographic assessment were performed. Trichoscope Aramo SG (x 60 and x 200), specialized diagnostic trichology software TrichoSciencePro $\odot$, camera Nikon 3100, stereotactic positioning device FOTOIMAGING were used.

\section{Status localis}

The lesions were located in vertex (predominantly) and occipital areas. The following clinical presentations were visualized: erythematous follicular papules, perifollicular erythema and perifollicular pustules, follicular hyperkeratosis, erosions, tufts and scaling, dilated, elongated vessels and hemorrhagic crusts (Figure 1a). Perifollicular pustules and abnormal vessels are presented on trichoscopy images - Figure $1 \mathrm{~b}$.

At the places of previously active lesions the small patches of cicatricial alopecia were observed, with milky-red and pinkish-white colour, and lack of follicular ostia (Figure 2a).

The active lesions were characterized by perifollicular erythema and pustules, erosions and yellowish scaling (Figure 2b).

From diagnostic point of view, the occipital lesion was of particular interest. The patient was complaining of strong itching and burning sensation in the area (Figure 3). Split and broken hairs in the area appeared due to intensive mechanical traumatization (scratching). The

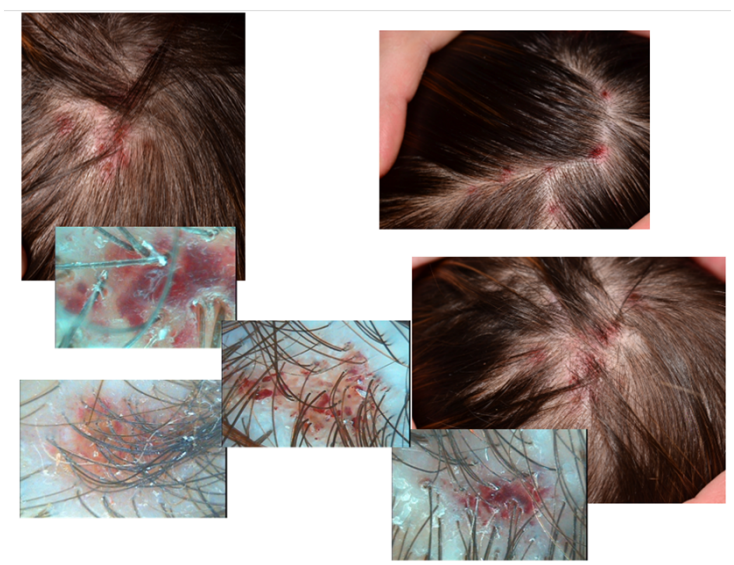

Figure 1a. Scalp condition of the patient at initial assessment (before treatment). Trichoscopy and global photographic assessment

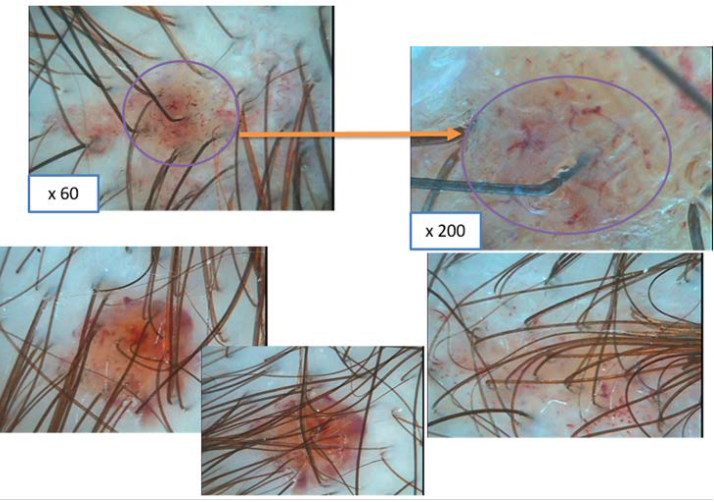

Figure $1 \mathrm{~b}$. Scalp condition of the patient at initial assessment (before treatment). Trichoscopy: Perifollicular pustules and Abnormal vessels (x 60 and x 200).

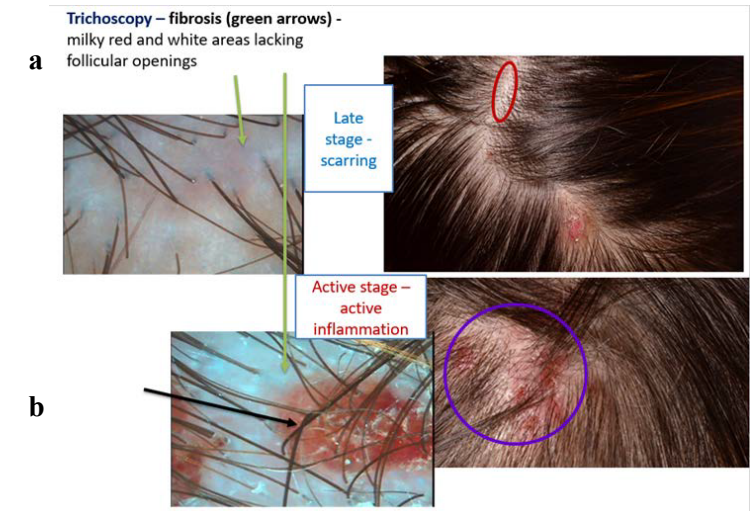

Figure 2. Scalp condition of the patient at initial assessment (before treatment). 2a. Late stage of FD - small areas of scarring alopecia (red oval). Milky-red and white areas are seen on trichoscopy images (green arrows). $2 \mathrm{~b}$. Active stage of FD - perifollicular erythema, erosions, yellowish scaling (black arrow) Both active and longstanding lesions are presented (in purple circle).

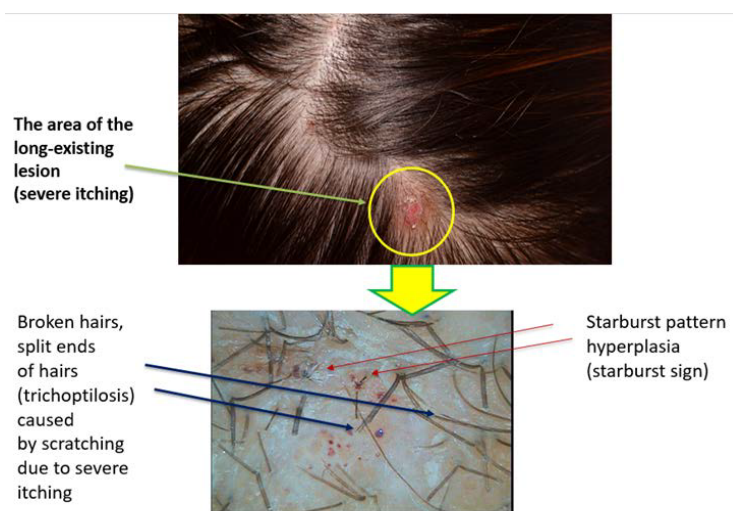

Figure 3. The occipital lesion of follicular decalvans with signs of scratching (in yellow circle).

Broken, split hairs (blue arrows), starburst pattern (red arrows).

starburst pattern hyperplasia (starburst sign), a characteristic feature of folliculitis decalvans, is visualized in this lesion.

\section{Clinical and laboratory diagnosis}

Laboratory tests Multisteroid analysis (blood test performed by liquid chromatography- mass spectrometry method) showed decreased levels of adrenal hormones: low level of corticosterone $=3.44$ (reference range $-3.8-66.5 \mathrm{nmol} / \mathrm{L}$ ), cortisol level at the bottom range $=158.5$ (reference range $-150-650 \mathrm{nmol} / \mathrm{L}$ ). Decreased cortisol level was also 
detected by saliva test.

The rheumatoid factor was increased, and the 25 hydroxy vitamin D (measured by liquid chromatography- mass spectrometry method) was decreased $=19$ (reference range $-50-100 \mathrm{ng} / \mathrm{ml}$ ). Staphylococcus aureus was detected in feces analysis and on the scalp lesions, the level of $S$. aureus on scalp lesions was $>2500 \mathrm{CFU} / \mathrm{dm}^{2}$ (normal range for scalp $-200 \mathrm{CFU} / \mathrm{dm}^{2}$ ). No biopsy was performed due to the patient's refusal.

\section{Diagnosis}

On the basis of the patient's complaints, medical history and physical examination the diagnosis was made: Folliculitis decalvans, relapsing course, acute stage.

\section{Treatment}

Taking into account inefficiency of preceding treatment and our previous successful experience of laser therapy in trichology patients [11], adjuvant therapy regimen was prescribed. The regimen included replenishment of deficient vitamin $\mathrm{D}$, nutrients and steroid hormones, as well as laser therapy and medical acupuncture treatment.

\section{Per oral therapy included:}

- Hydrocortisone - $15 \mathrm{mg}$ per day during 3 months, with subsequent gradual decrease of the medication and its further withdrawal;

- Vitamin D3 - 20000 IU per day, for 3 months, with subsequent decrease of the medication (under control of the levels of $25(\mathrm{OH})$ vitamin $\mathrm{D}$ and parathormone in blood, Calcium level in blood and urine).

Topical therapy included: Lotion with Epigallocatechin gallate, dipotassium glycyrrhizate, niacinamide, escine, vitamins, sulphur amino acids, pinadiol and camphanediol.

\section{Other adjuvant therapy}

Laser therapy: laser $662 \mathrm{~nm}+$ LED therapy $400 \mathrm{~nm}$ with lotion containing angioprotecting, anti-inflammatory components, antioxidants, moistening and sebum - regulating ingredients, № 12, 2 times per week, two courses.

Medical acupuncture treatment: № 15, 2 times per week, two courses.

Anti-inflammatory elimination rotation diet: products, containing dairy and gluten and some other products were excluded.

\section{Treatment results}

Adherence to the medication regimen brought the patient into remission. The patient also revealed decreased joints pain, bloating and epigastric pain, as well as decreased face and leg swelling. She lost about 11 kilograms of excessive weight.

\section{Status localis}

The lesions mainly resolved, with small patches of cicatricial alopecia remained, without wide-spreading of the disease (Figures 4 and 5). The pain and itching reduced, hair loss stopped.

Scalp condition significantly improved (Figure 6).

\section{Laboratory tests}

Multisteroid analysis (blood test performed by liquid

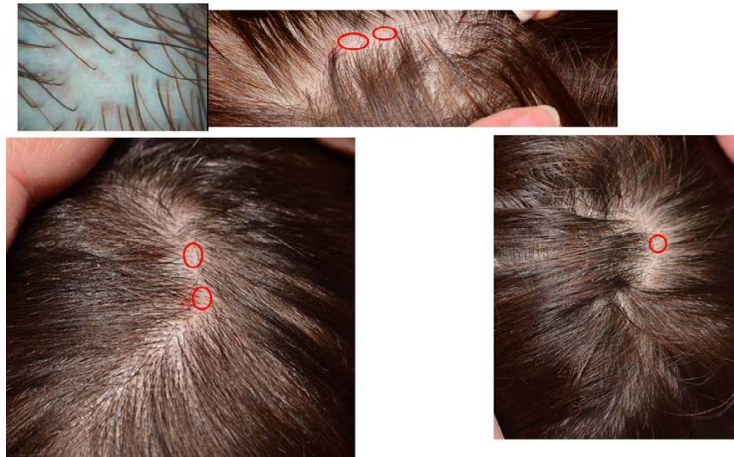

Figure 4. Trichoscopy and global photographic assessment.

After treatment: The small ivory - white patches of cicatricial alopecia remained as the outcome of scarring process (red circles).
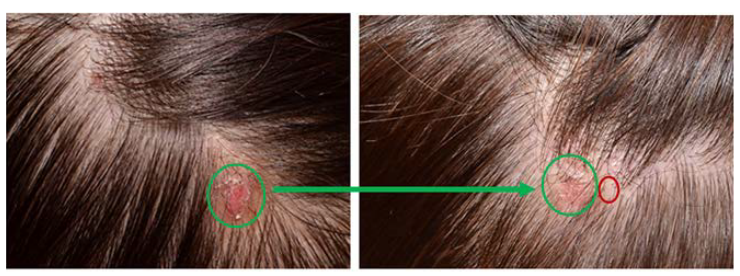

Figure 5. The longstanding lesion of FD (green circle): before and after treatment. The small patch of cicatricial alopecia remained as the outcome of scarring process (red circle).

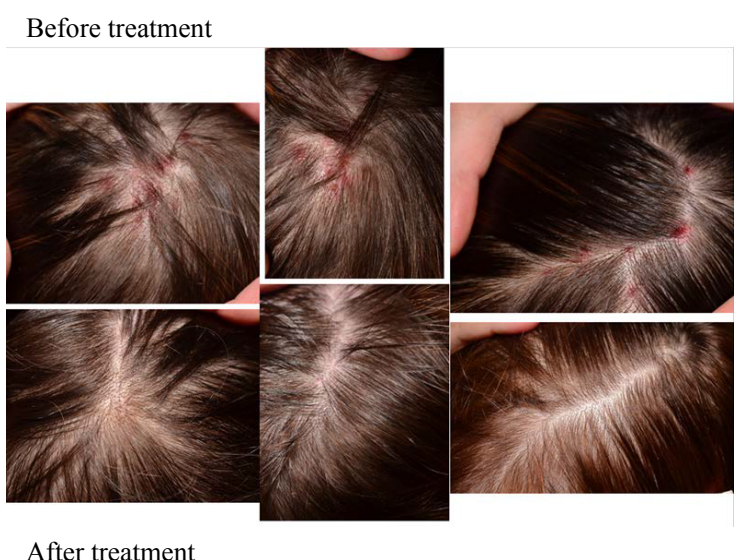

Figure 6. Before and after treatment.

chromatography- mass spectrometry method) showed increased levels of adrenal hormones: corticosterone from 3.44 to 11.70 (reference range - 3.8-66.5 nmol/L), cortisol level from 158.5 to 277.0 (reference range $-150-650 \mathrm{nmol} / \mathrm{L}$ ). The 25 hydroxy vitamin $\mathrm{D}$ (measured by liquid chromatography- mass spectrometry method) increased from 19.0 to 59.3 (reference range - $50-100 \mathrm{ng} / \mathrm{ml}$ ).

Limitations: no biopsy was performed due to the patient's refusal.

\section{Discussion}

The process of the current case of FD was prolonged and chronic, but the hair follicle destruction areas were not widespread, presenting a relatively mild form of Folliculitis decalvans.

The treatment was focused not on the suppression but on the recovery of the adrenal function, as physiological doses of hydrocortisone were used.

Relatively high doses of vitamin $\mathrm{D}$, known by their 
immunomodulating activity, were prescribed to increase the vitamin D level.

Topical treatment with adjuvant lotions and laser therapy helped to stop the inflammation in the scalp lesions and resolve the lesions.

Both medical acupuncture and anti-inflammatory diet helped to get not only local scalp improvement but also dramatically improved the overall patient's health, including her joints and gastrointestinal problems.

\section{Conclusion}

This clinical case shows the efficacy of integrative approach to a difficult trichology patient with folliculitis decalvans. The previous traditional treatment with antibiotics, topical glucocorticosteroids, oral prednisone and retinoid treatment had minor efficacy and subsequent recurrence.

Integrative approach to this difficult case included not only topical treatment, but also replenishment of deficient vitamin $\mathrm{D}$, nutrients and bioidentical steroid hormones. Anti-inflammatory diet, laser therapy and medical acupuncture treatment were used as well.

This regimen brought the patient into remission and improved the patient's scalp condition and overall health.

When we treat dermatology or trichology patients we usually focus just at their skin or scalp. And often we see only the tip of the iceberg. If we implement integrative approach, we look at the whole person, including his/her nutrition and whole well-being.

\section{References}

1. Olsen EA, Bergfeld WF, Cotsarelis G, Price VH, Shapiro J, et al. (2003) Summary of North American Hair Research Society (NAHRS)-sponsored Workshop on Cicatricial Alopecia, Duke University Medical Center, February 10 and 11, 2001. J Am Acad Dermatol 48: 103-110. [Crossref]

2. Blume-Peytavi U, Tosti A, Whiting D, Trueb R (2008) Hair growth and disorders Springer-Verlag. 208-210.

3. Andre MC, Soares RO. Effective Treatment of Folliculitis Decalvans: Azithromycin in Monotherapy. Hair Ther Transplant 5: 1

4. Tosti A (2007) Dermoscopy of hair and scalp disorders: pathological and clinical correlations. Informa Healthcare: 108-113.

5. Rudnicka L, Olszewska M, Rakowska A, Slowinska M (2011) Trichoscopy update 2011. J Dermatol Case Rep 5: 82-88. [Crossref]

6. Rudnicka L, Olzewska M, Rakowska A (2012) Atlas of Trichoscopy. Springer-Verlag, 2012: 319-329.

7. Annessi G (1998) Tufted folliculitis of the scalp: a distinctive clinicohistological variant of folliculitis decalvans. Br J Dermatol 138: 799-805. [Crossref]

8. Whiting DA (2001) Cicatricial alopecia: clinico-pathological findings and treatment Clin Dermatol 19: 211-225. [Crossref]

9. Petronic-Rosic V, Krunic A, Mijuskovic M, Vesic S. Tufted hair folliculitis: a pattern of scarring alopecia? J Am Acad Dermatol 1999: 41: 112-124.

10. Otberg N, Kang H, Alzolibani A, Shapiro J (2008) Folliculitis decalvans. Dermatologic Therapy 21: 238-244.

11. Khaldina M, Barunova N (2015) Low level laser therapy in trichology practice Trichology $J$ 1: 76-81.

Copyright: (C2016 Barunova N. This is an open-access article distributed under the terms of the Creative Commons Attribution License, which permits unrestricted use, distribution, and reproduction in any medium, provided the original author and source are credited. 